Review Article

\title{
Frequently Asked Questions (FAQs) about Coronavirus Disease 2019 (Covid-19)
}

Jugal Kishore', Shekhar Grover

${ }^{1}$ Director Professor \& Head, Vardhaman Mahavir Medical College, Safdarjung Hospital, New Delhi, India. ${ }^{2}$ Public Health Consultant, Maulana Azad Institute of Dental Sciences, New Delhi, India.

DOI: https://doi.org/10.24321/2455.7048.202004

\section{I $\quad \mathbf{N} \quad \mathbf{F} \quad \mathbf{O}$}

Corresponding Author:

Jugal Kishore, Vardhaman Mahavir Medical College, Safdarjung Hospital, New Delhi, India.

E-mail Id:

drjugalkishore@gmail.com

Orcid Id:

https://orcid.org/0000-0001-6246-5880

How to cite this article:

Kishore J, Grover S. Frequently Asked Questions

(FAQs) about Coronavirus Disease 2019

(Covid-19). Epidem Int 2020; 5(1): 14-21.

Date of Submission: 2020-04-02

Date of Acceptance: 2020-04-07
Currently, the entire world is battling with COVID-19 pandemic. India, too, has been affected by the disease. In the current state of crisis, it is pertinent to have appropriate knowledge about the disease. An attempt has been made to address some of the issues related to COVID-19. As the COVID-19 virus is novel, the evidence is evolving day by day. Yet, the information has been presented in the form of questions and answers with most updated and available evidence. (As on 6/04/2020).

\section{QI. What is COVID I9?}

Ans: COVID 19 stands for COrona VIrus Disease 2019. It is an infectious disease caused by novel strain of coronavirus, first detected in Wuhan, Hubei Province in China in December 2019. This new virus is named by International Committee on Taxonomy of Viruses (ICTV) as, "severe acute respiratory syndrome coronavirus 2 (SARS-CoV-2)" on 11th February, 2020.

\section{Q2. What is Coronavirus (Covid I9)?}

Ans: Coronaviruses are a large family of viruses that cause illnesses in animals and humans. In humans, coronaviruses cause infections ranging from common cold to severe lower respiratory tract infections. Severe Acute Respiratory Syndrome (SARS) in 2002-2003 and Middle East Respiratory Syndrome (MERS) in 2012 were also caused by coronaviruses.

\section{Q3. Where do Coronaviruses Come from?}

Ans: Bats are considered to be natural hosts for coronaviruses but some other animals also act as source of these viruses. For example, SARS-CoV- 1 is transmitted to humans from civet cats and MERS-CoV is transmitted from camels. However, the source for current outbreak due to SARS CoV-2 is not known. First infections were linked to live animal market in Wuhan but now there is human-to-human transmission.

\section{Q4. What are Modes of Transmission of SARS CoV-2?}

Ans: Initially when the infection began, virus seems to have been transmitted through animal source. But currently there is human-tohuman transmission.

The virus seems to be transmitted mainly via small respiratory droplets 
through sneezing, coughing, or when people interact with each other for some time in close proximity (usually less than one meter) while talking. These droplets may then be inhaled, or they land on surfaces. On touching these surfaces, one may come into contact with virus and contract infection on touching one's nose, mouth or eyes. The virus can thrive on various surfaces from several hours (copper: 8 hours, cardboard: 24 hours) up to a few days (plastic: 48 hours and stainless steel: 72 hours). However, the quantity of viable virus decreases over time and may not always be existing in adequate numbers to cause infection.

As of now, there is no evidence to suggest airborne transmission of COVID 19. One study has cultured the SARS CoV-2 from stool specimen. However, there is no evidence of fecal-oral route of transmission of COVID-19 virus, means transmitted from food or water is not established. Also, there is no evidence to suggest mosquito or tick borne transmission of the virus.

\section{Q5. What is Meant by Airborne Transmission and how is it Different from Droplet Transmission?}

Ans: Respiratory infections are usually transmitted by either respiratory droplets (>5-10 micrometer) or droplet nuclei ( $<5$ micrometer). Airborne transmission refers to the presence of microbes within droplet nuclei that can remain in air for longer period of time and usually transmitted to others over distance greater than 1 meter. Droplet transmission occurs when a person is in close contact (within 1 meter distance) of the person with respiratory symptoms. Transmission also occurs through fomites or objects used by the infected person.

In case of COVID-19, airborne transmission (via aerosols) may be possible while performing procedures for diagnostic and therapeutic purposes, such as laboratory procedures, endotracheal intubation, bronchoscopy, open suctioning, administration of nebulized treatment, manual ventilation before intubation, turning the patient to the prone position, disconnecting the patient from the ventilator, non-invasive positive-pressure ventilation, tracheostomy, and cardiopulmonary resuscitation. Evidence suggests virus remains stable in aerosols for 3 hours.

\section{Q6. What is the Risk of getting Infected with COVID- 19 from Animals or Animal Products from Affected Countries?}

Ans: Possible animal sources of COVID-19 are unknown. It is advisable to avoid visiting live animal markets, avoid direct contact with animals and surfaces in contact with animals. Further, it is advisable to practice healthy and safe food handling practices to avoid contamination of uncooked raw foods and animal products.

\section{Q7. Can I Catch COVID- I 9 from my Pet?}

Ans: There has been one instance of a dog being infected in Hong Kong. Similarly, a report has proved that a zoo tiger in New York was found positive for Covid 19. Therefore, it would be good decision to isolate the person from pets and domestic animal.

\section{Q8. What is the Risk of getting COVID-I9 Infection from Packages received through Online Shopping or Postal System?}

Ans: As discussed previously, there is some evidence that SARS CoV-2 survives on cardboard for 24 hours in experimental settings. However, in reality, there is no evidence of infection ever being transmitted through contaminated packages, as the virus is less likely to survive in transit owing to varying temperature and humidity conditions.

As precautionary step, some experts suggest that on receiving packages you can wipe the surface with disinfectant. Secondly, one can keep the entire package in some remote location or under the sun for some time (preferably for more than 24 hours) and then safely bring inside the home.

\section{Q9. What are Common Symptoms of COVID- I 9?}

Ans: Common symptoms of COVID-19 are dry cough, acute onset of high-grade fever, sore throat and difficulty in breathing. Symptoms vary in severity from having no symptom at all to having severe pneumonia, acute respiratory distress syndrome and septic shock.

Recently, anosmia (loss of sense of smell) and, in some cases, loss of sense of taste were also reported in some patients confirmed with SARS-CoV 2 infection in absence of other symptoms. $80.9 \%$ of infections are mild, $13.8 \%$ are severe while $4.7 \%$ require critical care.

\section{QI0. Who is at Risk of Developing Serious IIInesses with COVID-I9 Infection?}

Ans: COVID-19 is novel disease and there is limited information regarding its risk factors. However, evidence available till date suggests that older persons, persons with underlying medical conditions such as hypertension, diabetes mellitus, heart disease, lung disease and cancer may be at higher risk for developing serious illnesses. An immunocompromised person has higher risk of getting severe infection.

\section{QI I. What is Risk of Developing Severe Illnesses with COVID- I 9 Infection among Children and Pregnant Women?}

Ans: Children constituted very small proportion of COVID-19 cases with $1 \%$ under 10 years and $4 \%$ between 10 to 19 years. As per available evidence, they have lower risk of developing serious illnesses with COVID-19 infection. There is limited information regarding their potential to transmit the disease during asymptomatic or mildly symptomatic phase.

There is limited scientific evidence on the risk of developing severe illness among pregnant women with COVID-19 
infection. The clinical presentation among pregnant women is reported to be similar to non-pregnant women in China. Till date, no maternal mortality has been reported. There is no evidence for vertical transmission of SARS CoV-2. There is no evidence yet to suggest that SARS-CoV can be transmitted to neonate through breastfeeding. Therefore, it is advised that pregnant women infected with COVID-19 virus should continue breastfeeding by taking all droplet and contact precautions to prevent infecting the neonate.

\section{Q I 2. I have Recovered from Tuberculosis (TB), am I at Greater Risk of Getting Infected with COVID- 19 Virus?}

Ans: There is no data to suggest that patients who had history of TB are at greater risk of getting COVID-19 or worse outcomes (such as severe disease) with SARS CoV-2 virus infection. However, current status of active TB may increase the risk of severity of Covid 19 illness.

\section{Q I3. How would I Ensure Continuity of my Treatment for TB under Directly Observed Therapy (DOT) due to Social Distancing and National Quarantine Measures? \\ Ans: There exist various alternatives to DOT for treatment support to TB patients, which include Self-Administered Therapy (SAT) and support utilizing digital platforms such as Video-Observed Therapy (VOT) and other mobile phone- supported adherence strategies, such as 99DOTS. Under no circumstances the regular treatment of DOT should be stopped.}

Q I4. Are People Living with HIV/AIDS (PLHA) at Greater Risk of being Infected with COVID- 19 Virus?

Ans: PLHA with low CD4 counts and high viral loads and not taking treatment are at risk of contracting infections in general. Therefore, PLHA should take additional precautions such as timely intake of drugs, hand and personal hygiene and social distancing during this pandemic.

\section{Q I5. How can Government Assure Continuous access to HIV Services in Current Situation of Lockdown in India?}

Ans: There should be continuity of essential services (like multi-month prescriptions, distribution of condoms, needles and syringes and, may be, counseling sessions can decrease) pertaining to HIV/AIDs through drop in centres, outreach sessions and community-based models.

\section{Q I6. Is it True that Malaria Endemic Countries have been Protected against COVID- I9?}

Ans: There is no evidence to suggest that malaria endemic countries have been protected against COVID-19. According to $\mathrm{WHO}$, malaria-endemic countries in all WHO have regions have reported cases of COVID-19.

\section{Q I7. How many Countries are Free from} COVID-19?

Ans: COVID-19 has affected 208 countries across the world as on $5^{\text {th }}$ April, 2020. Following countries have not reported a single case: North Korea, Solomon Islands, Tajikistan, Nauru, Yemen, Kiribarti, Marshall Islands of Palau, Federated States of Micronesia, Samoa, Micronesia, Turkmenistan, Tuvalu, Vanuatu, Comoros and Tongo.

Q I8. What is the Case Fatality Rate (CFR) of COVID-I9 in Comparison to other Coronaviruses and Influenza?

Ans: Globally approximately 3.4\% of reported COVID-19 cases have died. The CFR of COVID-19 appears to be lower than that of SARS (9.5\%) and MERS (34.4\%), but higher than that of influenza $(0 \cdot 1 \%)$.

Q19. What is the Incubation Period of COVID-19? When a Person is Considered to be Infectious?

Ans: Incubation period refers to time period between entry of an infectious agent in host body to development of symptoms. In context of COVID-19, it is considered between 2 to 14 days, most commonly around 5 days. However, a person may transmit the infection 24 to 48 hours before the symptomatic phase and last for 7 to 12 days in case of moderate infection and 14 days in severe cases.

\section{Q20. What is Transmissibility Potential of} COVID-I 9 Virus?

Ans: Virus transmissibility is measured by quantity referred as basic reproductive number or $R_{0}$. $R_{0}$ refers to average number of people who will catch a disease from a contagious person. In context of COVID-19, it is considered to be between 2 to 2.5 .

(Note: Three possibilities exist for the potential spread or decline of a disease, depending on its $R_{0}$ value: 1 . If $R_{0}$ is less than 1 , each existing infection causes less than 1 new infection. In this case, the disease will decline and eventually disappear. 2. If $R_{0}$ equals 1 , the disease will stay alive, but there won't be an epidemic. 3. If $R_{0}$ is greater than 1 , cases could grow exponentially and cause an epidemic or even a pandemic. Our aim should be to bring the $R_{0}$ to less than 1 ).

Q2I. What is a Cluster and Hotspot in COVID-I 9 Epidemic?

Ans: Cluster refers to an aggregation of cases grouped in place and time that are suspected to be greater than the number expected, even though the expected number may not be known. In context of COVID-19, areas with more than 3 infections (epidemiologically linked) have been identified as clusters.

"Hotspot" is frequently used to refer to areas of elevated 
disease burden or high transmission efficiency. They are also called spatial clusters. In context of COVID-19, hotspot has been referred to an area with more than 10 cases.

\section{Q22. What do you mean by Community Transmission of SARS CoV-2?}

Ans: Community transmission of virus means that people have been infected with virus in an area with some people not sure as to how and when they got infected.

\section{Q23. What is Serial Interval of COVID-I 9 Virus?}

Ans: Serial interval refers to time between successive cases. The serial interval for COVID-19 virus is estimated to be 5 to 6 days, while for influenza virus, the serial interval is 3 days.

\section{Q24. What Temperature Kills the Virus that Causes COVID-19?}

Ans: Generally, coronaviruses do not survive for longer periods at high temperatures and high humidity when compared to cooler and dry climatic conditions $\left(R_{0}\right.$ value $>2$ ). It is estimated that the $R_{0}$ value decreases to 1.09 at the temperature of $40^{\circ} \mathrm{C}$. As the temperature reaches $43^{\circ} \mathrm{C}$ the $R$ value might reduce to even less than 1

\section{Q25. How can I Protect myself from COVID- I9 Infection?}

Ans: You can protect yourself from getting infected by practicing following:

- Wash your hand with soap and water for at least 20 seconds or cleaning hands thoroughly with alcohol based solutions.

- Do not touch your eyes, nose or mouth with unwashed hands.

- It is recommended to stay more than 1 meter away from people infected with COVID-19 infection.

- Try to stay indoors/at home as much as possible. Avoid non-essential travel particularly to affected areas.

- Cover your mouth/nose while coughing or sneezing.

To summarize, one should practice cough etiquette, personal hygiene and maintain social distancing to avoid getting infected with COVID-19 virus.

\section{Q26. When and who Should Wear Mask?}

Ans: Mask should be worn only:

- When a person develops cough or fever

- While visiting a healthcare facility

- While caring for an ill person with COVID-19

- Close family contacts of such suspect/confirmed cases undergoing home care

In all the above instances, triple layer medical mask can be worn (effective for 8 hours).

For extra precautions, the Government of India has issued advisory for use of home-made protective cover for face and mouth to be used by those people who are healthy (not suffering from medical conditions or breathing difficulties), particularly when they step out of house.

\section{Q27. What is Social/ Physical Distancing? How does it Prevent Spread of COVID-19?}

Ans: Social distancing is maintaining distance of more than 1 meter from their social contacts-family, friends and colleagues. This aims to reduce physical contact between potentially infectious patients with healthy people thereby decreasing or interrupting the transmission of virus. It is helpful in all droplet infections, such as H1N1, SARS, MERS, etc. Since COVID-19 is droplet-borne infection, social distancing is an effective strategy for prevention of spread in the community. Without it the spread of infection would be very high and will create sharp hike epidemic curse. But social distancing will decrease the spread of infection in community and helps to "flatten" the epidemic curve.

Some of the social distancing measures taken by the Indian government are:

- Closure of all educational institutions

- Closure of shopping malls, cinema halls and shopping complexes

- Postponement of examinations

- Encouraging public and private sector employees to work from home

- Avoiding mass gatherings and rescheduling of conferences, workshops and meetings

- Postponement of all non-essential social and cultural gatherings

- Postponement of all sporting events

- Non-essential travel to be avoided

Q28. What is Lockdown in Context of COVID-I9 Pandemic in India?

Ans: A lockdown is an emergency public health protocol that prevents people from leaving a given area. A full lockdown will mean you must stay where you are and not exit or enter a building or the given area. This ensures that infection will not spread from one person to another as there is no movement of people.

This scenario usually allows for essential supplies, grocery stores, pharmacies and banks to continue to serve the people. The entire country is under lockdown since $25^{\text {th }}$ March, 2020 to $14^{\text {th }}$ April, 2020.

\section{Q29. What to do if I had a Contact with a Patient with COVID- I9?}

Ans: A contact is a person who is involved in any of the following:

- Providing direct care without proper Personal Protective 
Equipment (PPE) for COVID-19 patients,

- $\quad$ Staying in the same close environment of a COVID-19 patient (including workplace, classroom, household, gatherings)

- Traveling together in close proximity $(1 \mathrm{~m})$ with a symptomatic person who later tested positive for COVID-19

\section{High-risk Contact}

- Touched body fluids of the patient (respiratory tract secretions, blood, vomit, saliva, urine, feces)

- Had direct physical contact with the body of the patient including physical examination without PPE

- Touched or cleaned the linens, clothes, or dishes of the patient

- Lives in the same household as the patient

- Anyone in close proximity (within 1 meter) of the confirmed case without precautions

- Passenger in close proximity (within 1 meter) of a conveyance with a symptomatic person who later tested positive for COVID-19 for more than 6 hours

\section{Low-risk Contact}

- $\quad$ Shared the same space (same class for school/worked in same room/similar and not having a high-risk exposure to confirmed or suspect case of COVID-19).

- Travelled in same environment (bus/train/flight/any mode of transit) but not having a high-risk exposure.

Since data is insufficient to precisely determine the duration of exposure* and closeness of contact, a person coming in contact with COVID-19 patient, should undergo selfquarantine for 14 days (preferably at home) beginning from day of contact and self-monitor for symptoms of COVID-19 infection. In case, one observes any of the symptoms mentioned above, contact nearest health facility for further advice and management.

(*Note: Recommendations vary on duration of exposure from 10 minutes or more to 30 minutes or more. In healthcare settings, it is considered as any exposure greater than few minutes).

\section{Q30. When should I be tested for COVID- I9?}

Ans: The current testing strategy in India is as follows:

- All asymptomatic individuals who have undertaken international travel in the last 14 days and have become symptomatic

- All symptomatic contacts of laboratory confirmed cases

- All symptomatic healthcare workers

- All hospitalized patients with Severe Acute Respiratory Illness (fever AND cough and/ or shortness of breath)

- Asymptomatic direct and high-risk contacts of a confirmed case should be tested once between day 5 and day 14 of coming in his/ her contact

\section{Q3 I. If I am a Candidate for Testing where Should I go?}

Ans: There are 126 government and 51 private laboratories across the country, which have been approved by Indian Council of Medical Research for COVID-19 testing. The details of these laboratories can be accessed at: https:// www.icmr.nic.in/node/39071

Q32. What is gold standard tool for diagnosis of COVID- I 9? When, during infection, are they effective? What samples are needed for testing?

Ans: Real Time Reverse Transcriptase-Polymerase Chain Reaction (rtRT-PCR) is currently being used to confirm the diagnosis of COVID-19. RT-PCR in combination with Computed Tomography (CT) scan is the most effective with sensitivity of $91.1 \%$. RT-PCR has been able to detect COVID-19 virus in 2/114 individuals who were asymptomatic.

The most common sample types being tested are swabs taken from the nasopharynx and/or oropharynx, with the former considered somewhat more sensitive than the latter. For patients with pneumonia, in addition to nasopharyngeal and oral secretions, lower respiratory tract secretions, such as sputum and bronchoalveolar lavage fluid, are tested.

\section{Q33. What are the drug or vaccine available for prevention and treatment of COVID-19?}

Ans: As of now, there is no approved drug or vaccine for prevention or cure of COVID-19 infection. Hydroxychloroquine has been recommended as chemoprophylaxis drug for use by asymptomatic healthcare workers managing COVID-19 cases and asymptomatic contacts of confirmed COVID-19 cases. In addition, a combination of Hydroxychloroquine and Azithromycin has been advocated for use in severe cases of COVID-19 under medical supervision.

\section{Q34. Does vaccination with BCG provide any protection against COVID-19?}

Ans: BCG vaccine helps to boost the innate immune response and prevent infective pathogens from entering the human body. There is no data to suggest effect of BCG on coronaviruses in general or SARS CoV-2 in particular. However, there is hypothesis that that countries without universal policies of BCG vaccination (Italy, Nederland, USA) have been more severely affected compared to countries with universal and long-standing BCG policies. Countries that have a late start of universal BCG policy (Iran, 1984) had high mortality, consistent with the idea that BCG protects the vaccinated elderly population. More studies are required to establish any beneficial effect of BCG.

Q35. What are the initiatives taken by Government of India? From where authentic information can be obtained?

Ans: Government of India has taken many commendable 
initiatives to combat COVID-19 pandemic. Some of the initiatives are:

- $\quad$ Dedicated 24 by 7 helpline number: +91-11-23978046 \& Toll Free number: 1075; Helpline email ID: ncov2019@ gov.in

- Dedicated dashboard for coronavirus updates

- Isolation, quarantine, strict surveillance and testing protocols for COVID-19

- Online Trainings for health workforce across the country through telemedicine

- National lockdown for 21 days and social distancing implementation to prevent stage 3 of epidemic and flattening of epidemic curve.

- Financial package for migrant workers and poor vulnerable population; life insurance cover for health workers

- Aarogaya Setu App: for information and alert on COVID-19

- Guidelines for psychosocial care, chemoprophylaxis for health workers, dead body management already formulated

- Fact check at Press Information Bureau to prevent spread of fake news regarding COVID-19

- Behavioral psychosocial helpline: Toll free number-08046110007

It is worth mentioning that national government has worked pro-actively to prevent the spread of disease in the country.

\section{Myths related to COVID-19}

Taking Honey, tulsi, ginger, lemon daily will prevent the person from Covid 19!

Ans: These home remedies are not going to prevent the person from Covid-19. However, Ministry of AYUSH has given press release for using drinking warm water, haldi (turmeric) milk, chayavanprash, herbal tea, Munakka, etc. Still they have clearly indicated that these all advises do not treat the COVID-19 but enhance immunity.

\section{Garlic boosts the immunity system}

Ans: This is a myth. There is no scientific evidence to suggest that the consumption of garlic boosts the immunity system or safeguards one from being infected with COVID-19. However, garlic is a healthy food that may have some antimicrobial properties.

Everyday Vitamin C will boost immune system and protect from COVID-19

Ans: Vitamin $C$ is an essential nutrient that can support immune function. On daily basis, Vit $\mathrm{C}$ should be part of our balanced diet. However, there is no evidence from the current outbreak that eating citrus fruits has protected people from the new coronavirus.
Eating meat and chicken will cause one to have COVID-19

Ans: This perception is due to linking origin of corona virus (COVID-19) with sea-food market in Wuhan but now infection is transmitted person to person through droplets, and well-cooked meat or chicken is safe to eat.

Taking hot water bath will destroy corona virus?

Ans: No, hot water bath may not be killing the pathogen inside the body. Hot water more than $60^{\circ} \mathrm{C}$ required to kill virus but at this temperature it is difficult to take bath. Warm water with soap is good for cleaning the body during bath that helps to keep our body hygiene.

Gargle with salt water regularly can help prevent infection with the COVID-19 as well as drinking water to wash off the viruses from the throat

Ans: False. There is no evidence that regularly gargle with hot water has protected people from infection with the new coronavirus. Gargles with warm water may help soothe a sore throat, this practice will not prevent the virus from entering your lungs-neither will drinking frequent sips of water.

\section{Sitting under the Sun will prevent COVID-19}

Ans: This is false belief. There is no evidence that the sunlight can kill COVID-19 inside the body. Getting enough sunlight may have other health benefits. For example, sunlight is good to get Vitamin $D$, but too much exposure to sunlight may also lead to sunburn. Exposure to sunlight has disinfectant property on surfaces such as tables, chair, doors, etc.

Spraying alcohol or chlorine all over the body can kill the Corona virus

Ans: False. Spraying alcohol or chlorine all over your body will not kill viruses that have already entered your body. Spraying such substances can be harmful to clothes or mucous membranes (i.e. eyes, mouth). Be aware that both alcohol and chlorine can be useful to disinfect surfaces, but they need to be used under appropriate concentration and recommendations.

Drinking whiskey or wine or any alcohol will kill corona viruses.

Ans: No alcohol drink can kill COVID-19. Rather they are going to destroy liver and pancreas. Immunity goes down in people who take alcohol further increasing the risk of COVID-19.

Chinese people are bad who have endangered whole world

Ans: This is wrong perception about any Chinese people because disease pathogen can originate from anywhere in the world. For example, Swine Flu originated from Mexico in 2009; MERS corona virus originated from Saudi Arabia 
in 2012. Ebola is a highly fatal disease that emerged from southeastern Guinea, Africa in 2014. In last century, 20 million people died due to Spanish Flu, therefore, time to time infectious diseases keep emerging or re-emerging and targeting human race. We all have to work together to prevent them and fight against them united.

\section{Mosquito bites can fast track transmission}

Ans: This is a myth. No study or research has shown that mosquitoes can act as carriers of the novel coronavirus. "The new coronavirus is a respiratory virus which spreads primarily through droplets generated when an infected person coughs or sneezes, or through droplets of saliva or discharge from the nose," says WHO.

\section{Hand dryers/ ultraviolet disinfection lamp can kill the virus}

Ans: These are myths. According to the World Health Organisation (WHO), one should regularly wash one's hands with soap and water for 20 seconds. Apart from this, neither a hand dryer nor an ultraviolet disinfection lamp can kill the virus.

\section{Vaccines against pneumonia protect against novel coronavirus}

Ans: This is a myth. Pneumococcal vaccine and Haemophilus influenza type $B(\mathrm{Hib})$ vaccine cannot protect anyone from COVID-19 since the vaccine for the new coronavirus is yet to be discovered.

\section{Parcels from China can spread COVID-19}

Ans: This is untrue. While some preliminary research has shown that the novel coronavirus remains on certain surfaces such as cardboard for some time, it cannot spread through parcels received from China.

\section{Thermal scanners can detect novel coronavirus}

Ans: This is not entirely true. A thermal scanner detects body temperature and is great for identifying someone with a high fever; however, medical experts and doctors treating Covid-19 patients have said that initial research shows how people who display no symptoms can also be infected with the virus.

\section{Holding breath for 10 seconds acts as test for COVID-19}

Ans: This is a myth. Dr Faheem Younus, the Chief of Infectious Diseases at the University of Maryland says that a person infected with the novel coronavirus can also hold his/her breath for longer than 10 seconds. On the other hand, the elderly will not be able to accomplish this task but it does not in any way mean that they are infected.

Temperature, humidity could affect transmission rate of COVID-19

Ans: This is a myth. The World Health Organisation (WHO) has observed that this infection can be transmitted in any and all areas regardless of climate.

\section{Wearing a mask can prevent transmission of COVID-19}

Ans: This is not true. While wearing a mask in a crowded place is advisable, the mask cannot prevent transmission of the novel coronavirus. In some cases, where the person wearing the mask touches an infected surface before touching his/her face to re-adjust the mask, this protective gear could lead to infection.

\section{Novel coronavirus affects only elderly, not young people}

Ans: This is untrue. The transmission of novel coronavirus has been seen in people of all ages. Doctors suggest that people with comorbid conditions such as blood pressure, heart or respiratory diseases are at a higher risk if they get infected with COVID-19. However, a perfectly healthy person can also be infected in the presence of a COVID-19 patient.

\section{Acknowledgement}

Dr. Tanu Anand, Scientist D, DHR, ICMR has greatly contributed in preparing this manuscript.

\section{Conflict of Interest: None}

\section{References}

1. WHO. Q \& A on Coronaviruses (COVID 19) [Internet] 2020 [Accessed on $31^{\text {st }}$ Mar, 2020]. Available from: https://www.who.int/news-room/q-a-detail/q-acoronaviruses.

2. MoHFW. COVID-19 India Updates [Internet] 2020 [Accessed on $4^{\text {th }}$ April, 2020]. Available from: https:// www.mohfw.gov.in/index.html\#.

3. Worldometer. COVID-19 Coronavirus pandemic [Internet] 2020 [Accessed on $4^{\text {th }}$ April, 2020]. Available from: https://www.worldometers.info/coronavirus/.

4. ICMR. COVID 19 [Internet] 2020 [Accessed on $4^{\text {th }}$ April, 2020]. Available from: https://www.icmr.nic. in/node/39071.

5. CDC. Coronavirus 2019 (COVID-19) Frequently Asked Questions [Internet] 2020 [Accessed on $4^{\text {th }}$ April, 2020]. Available from: https://www.cdc.gov/ coronavirus/2019-ncov/faq.html.

6. ECDC. Q \& A on COVID 19 [Internet] 2020 [Accessed on $4^{\text {th }}$ April, 2020]. Available from: https://www.ecdc. europa.eu/en/novel-coronavirus-china/questionsanswers.

7. DHS Science \& Technology Master Question List for COVID-19 (Caused by SARS CoV-2) [Internet] 2020 [Accessed on $4^{\text {th }}$ April, 2020]. Available from: https:// www.dhs.gov/sites/default/files/publications/mql_ sars-cov-2_-cleared-for-public-release_2020_03_25. pdf.

8. Miller A, Reandelar MJ, Fasciglione K, Roumenova 
V, Li Y, Otazu GH. Correlation between universal BCG vaccination policy and reduced morbidity and mortality for COVID-19: an epidemiological study. MedRxiv 2020; https://doi.org/10.1101/2020.03.24.20042937.

9. NCDC. Disease Alerts: Novel Coronavirus 2019 [Internet] 2020 [Accessed on $4^{\text {th }}$ April, 2020]. Available from: https://ncdc.gov.in/index4. php?lang=1\&level=0\&linkid=127\&lid $=432$.

10. https://www.livemint.com/news/india/coronavirusdisease-clusters-emerge-as-new-battle-front-in-waragainst-covid-19-11585680593968.html.

11. https://economictimes.indiatimes.com/magazines/ panache/in-a-first-bronx-zoo-tiger-nadia-tests-positivefor-covid-19-develops-dry-cough-loss-of-appetite/ articleshow/75001865.cms. 